\title{
The Expression of apxIVA Gene of Actinobacillus pleuropneumoniae Serotype 5 and Establishment of an Indirect ELISA for Distinguishing Infection and Immunization
}

\author{
Nianli Zou ${ }^{1}$, Yulan Lai ${ }^{1}$, Min $\mathrm{Li}^{1}$, Sanjie Cao ${ }^{1,2}$, Xintian Wen ${ }^{1,2}$ and Yong Huang ${ }^{*}, 1,2$ \\ ${ }^{1}$ College of Veterinary Medicine, Sichuan Agricultural University. Ya'an 625014, China \\ ${ }^{2}$ Key laboratory of Animal Disease and Human Health of Sichuan Province, Sichuan Agricultural University, Ya'an \\ 625014, China
}

\begin{abstract}
Actinobacillus pleuropneumoniae is the etiological agent of Porcine pleuropneumonia, which causes severe losses in pig farming. To establish an serological method to detect $A$. pleuropneumoniae infection, bioinformatics method was utilized to analyzed the sequence of apxIVA gene of $A$. pleuropneumoniae serotype 5 completed by our lab, a $1152 \mathrm{bp}$ fragment of the N'-terminal of apxIVA gene was amplified and cloned into the prokaryotic expression vector pET-32a(+), a recombinant protein about $62 \mathrm{KD}$ was expressed upon isopropy $1-\beta-\mathrm{D}$ - thiogalactoside (IPTG) induction. The protein could react specifically with antiserum from live A. pleuropneumoniae infection in western-blot. After further purification by Ni-NTA, this protein was used to establish an indirect ELISA to detect A. pleuropneumoniae infection. This method showed high specificity and could react positively with antibodies of live A. pleuropneumoniae infection while negatively with those of inactivated A. pleuropneumoniae immunization. In conclusion, this indirect ELISA could be used to detect A. pleuropneumoniae infection in mice model.
\end{abstract}

Keywords: Actinobacillus pleuropneumoniae, ApxIVA, serotype 5, indirect ELISA, infection, immunization.

Porcine pleuropneumonia is an acute or chronic respiratory disease characterized by hemorrhagic, fibrinous, and necrotic lung lesions, and A. pleuropneumoniae is the etiological agent [1]. The disease is highly contagious and associated with all ages of growing pigs, often co-infecting with other pathogens such as Hog cholera virus, Porcine reproductive and respiratory syndrome virus and Pasteurella multocida etc, resulting in severe economic losses in swine industry. The morbidity ranges from $8.5 \%$ to $100 \%$ while the fatality ranges from $0.4 \%$ to $100 \%$. A pleuropneumoniae infection is more prevalent under intensive breeding conditions. To date, 15 serotypes have been reported with serovars 1, 7, and 12 making up approximately $90 \%$ of isolates $[2,3]$.

A. pleuropneumoniae-RTX toxins (apx) is the main virulence factor and protective antigen of $A$. pleuropneumoniae. To update, four kinds of apx toxins were found produced by $A$. pleuropneumoniae, named apx I, apx II, apxIII and apxIV respectively [4]. The apx toxins produced by $A$. pleuropneumoniae varied with serotypes, but all serotypes can secrete apxIV which can be produced only during infection, but not under in vitro conditions [4]. ApxIV can also induce specific antibodies of high titres [5]. Based on the above character, bioinformatics method was used to analyzed the apxIVA gene sequence of serotype 5 completed by our lab, and a $1152 \mathrm{bp}$ long fragment of the N'-terminal of apxIVA gene of serotype 5 was cloned and expressed to

*Address correspondence to this author at the College of Veterinary Medicine, Sichuan Agricultural University. Ya'an 625014, Sichuan, China; Tel: +868352886117; Fax:+868352886117; E-mail: hyong601@163.com establish an indirect ELISA for detecting $A$. pleuropneumoniae infection in mice model.

\section{MATERIALS AND METHODOLOGY}

\section{Bacterial Strain and Grown Conditions}

E. coli JM 109, DH5a and BL21 (DE3) were purchased from Invitrogen corporation (California, USA) and cultured in LB, A. pleuropneumoniae serotype 1 strain CVCC 259 and serotype 5 strain CVCC 263 and serotype 7 strain CVCC 265 were purchased from China institute of Veterinary Drug Control (Beijing, China) and proliferated in Tryptic Soy Broth (TSB) with $0.001 \%$ nicotinamide adenine dinucleotide (NAD).

\section{Experiment Mice}

Female Kun-Ming mice (six weeks old, 17-22 g), purchased from the experimental animal center of Sichuan University (Chengdu, China), were maintained in animal holding laboratory under controlled condition with temperature of $25 \pm 10^{\circ} \mathrm{C}$, humidity of $40 \pm 10 \%$ and had free access to standard mouse diet and water.

\section{Sera}

For the production of mice antisera against live or inactivated $A$. pleuropneumoniae serotype 5 , the $50 \%$ of lethal dose (LD50) were determined firstly. Six-week-old Kun-Ming mice were randomly divided into five groups, each of them consisting of eight mice. and injected intraperitoneally (i.p.) with $200 \mu \mathrm{l}$ TSB containing $A$. pleuropneumoniae $\left(1.0 \times 10^{8}\right.$ to $1.0 \times 10^{4} \mathrm{CFU} /$ mouse). The number of surviving mice was recorded 5 days after 
infection. LD50 was calculated by the method of Reed and Muench and determined to be $6.3 \times 10^{6}$ CFU. Then Sixweek-old Kun-Ming mice were randomly divided into two groups, each of which consisting of twelve mice. Mice in group A were injected i.p. with $200 \mu$ of $A$. pleuropneumoniae preparation containing $6 \times 10^{5} \mathrm{CFU}$ per mouse on days 0,14 and 28 respectively; while mice in group B were inoculated i.p. with the same amount of inactivated A. pleuropneumoniae as group A per mouse on days 0,14 and 28 respectively. Mice were bled from eyes 10 days following the final boost and sera were collected. For the production of mice antisera against live $A$. pleuropneumoniae serotype 1 or 7 , twelve mice were inoculated with live $A$. pleuropneumoniae preparation containing 1/10 LD50 per mouse following the method above.

\section{Construction of Recombinant Prokaryotic Expression Vector}

A. pleuropneumoniae present in $1 \mathrm{ml}$ of an overnight culture were collected by centrifugation and DNA was extracted using a sodium dodecyl sulfate (SDS) - proteinase $\mathrm{K}$ and phenol/chloroform protocol as described in previous research [6]. Then, bioinformatics method was used to analyze the apxIVA gene sequence of A. pleuropneumoniae serotype 5 completed by our lab (Genbank NO: GQ332268), a pair of specific primers was used to amplify the $1152 \mathrm{bp}$ fragment of the 5'-terminal of apxIV A gene. The two primers were 5'CCA GAATTC ACG TTA GAC GAA ACA AGG- 3' with EcoRI site (underlined) comprising position 655-672 of apxIVA coding sequence and 5'CTC CTCGAG TTA ATT CAG ATC GGA GGC A -3' with XholI site (underlined) comprising position 1791-1806 of apxIVA coding sequence. The oligonucleotides were synthesized by Takara biotechnology (Dalian, China) co., ltd. Amplification of the apxIVA gene was performed with polymerase chain reaction (PCR) and the PCR products were cloned into the pMD18-T plasmid (Takara) to yield recombinant plasmid. Confirmation of clones containing recombinant plasmid was achieved by PCR and restriction enzyme (RE) digestion, and the correct clones were sequenced by Takara biotechnology (Dalian). The recombinant plasmid were then digested with EcoRI and XholI and cloned into pET-32a(+) (Novagen, Madison, USA) digested with EcoRI and XholI to generate recombinant prokaryotic expression plasmid.

\section{Expression and Purification of apxIVA}

The BL21 strains containing the recombinant prokaryotic plasmid were grown overnight with shaking at $37^{\circ} \mathrm{C}$. The overnight cultures were diluted in the proportion of $1: 100$ and incubated at $37^{\circ} \mathrm{C}$ with vigorous shaking for 3-4 hours (h.). The production of poly-His-tailed ApxIV a proteins were induced by addition of $1 \mathrm{mM}$ IPTG and incubation for a further 3-4 h. The cells were harvested and analyzed by sodium dodecyl sulfate polyacrylamide gel electrophoresis (SDS-PAGE). Optimization of expression conditions at different IPTG concentration ranging from $0,0.2,0.4,0.6$, $0.8,1.0$ to $1.2 \mathrm{mM}$ and different induction time ranging from $0,2,3,4,5$ to $6 \mathrm{~h}$ were also conducted.

For purification of recombinant apxIVA, the cells were harvested from liquid cultures by centrifugation and suspended in phosphate-buffered saline (PBS) ( $\mathrm{pH} 7.2$ ) containing lysozyme $(1 \mathrm{mg} / \mathrm{ml})$ by $1 / 10(\mathrm{v} / \mathrm{v})$, after ice bathing for $30 \mathrm{~min}$, the suspension were sonicated and centrifuged at $12000 \mathrm{~g}$ for $10 \mathrm{~min}$. The pellets were homogenated and washed with washing buffer [50 mM TrisHcl (PH8.0), 1 mM EDTA, 0.2\% TritonX-100, $2 \mathrm{M}$ urea] for three times at $10 \mathrm{~min} /$ times and centrifugated at $12000 \mathrm{~g}$ for $10 \mathrm{~min}$. The pellets were dissolved by denaturation buffer [50 mM Tris-Hcl (PH8.0), 2 mM 2-mercaptoethanol, $8 \mathrm{M}$ urea] and supernatant were collected after centrifugation. The supernatant was treated with renaturation buffer $[50 \mathrm{mM}$ Tris-Hcl (PH8.0), $0.1 \mathrm{mM}$ oxidized glutathione, $1 \mathrm{mM}$ reduced glutathione, $0.5 \mathrm{M}$ urea] for overnight at $4{ }^{\circ} \mathrm{C}$ and filtrated through $0.45 \mu \mathrm{M}$ filtration membrane. Then the solution was purified on a column packed with Ni-NTA His.Bind superflow according to the manufacture's instruction (Merck, Darmstadt, Germany). The eluate from each imidazole concentration were collected and analyzed by SDS-PAGE.

\section{Western Blot Analysis}

Purified proteins were separated by SDS-PAGE, then the proteins were transferred to nitrocellulose membrane with $0.45 \mu \mathrm{m}$ pore size (Millipore Corp., USA) at $15 \mathrm{~V}$ for $1.5 \mathrm{~h}$ with a Bio-Rad Transblot Cell as described in the manufacturer's specifications. The membrane was then blocked for $90 \mathrm{~min}$ with milk buffer $(20 \mathrm{mM}$ Tris- $\mathrm{HCl} \mathrm{pH}$ $8.0,150 \mathrm{mM} \mathrm{NaCl}, 0.05 \%$ Tween $20,5 \%$ skimmed dry milk) at $37^{\circ} \mathrm{C}$, and then washed with Tris-buffered saline with Tween 20 (TBST) buffer (20mM Tris- $\mathrm{HCl} \mathrm{PH} \mathrm{8.0,} \mathrm{150mM}$ $\mathrm{NaCl}, 0.05$ Tween-20) for three times and incubated with mouse antiserum diluted $1: 100$ in $0.5 \%$ bovine serum albumin (BSA) $/ \mathrm{PBS}$ for $60 \mathrm{~min}$ at $37^{\circ} \mathrm{C}$. The membrane was washed with TBST, then incubated with horseradish peroxidase (HRP) -labeled sheep-anti-mouse IgG (zhongshan Goldenbridge Biotechnology Co., LTD, Beijing, China) for $60 \mathrm{~min}$ at $37^{\circ} \mathrm{C}$. Target proteins were visualized using 3,3'- diaminobenzidine (Tiangen, Beijing, China).

\section{Development of an apxIVA-ELISA}

A 96-well microtiter plate (Costar, USA) was coated with $100 \mu \mathrm{l}$ of purified apx IVA and incubated at $4^{\circ} \mathrm{C}$ overnight. The plate was then blocked for 90 min with milk buffer $(5 \%$ skimmed dry milk in PBS) at $37^{\circ} \mathrm{C}$ and washed twice with PBS. Subsequently, $100 \mu \mathrm{l}$ of mice sera were added and incubated at $37^{\circ} \mathrm{C}$ for $60 \mathrm{~min}$. The samples were washed, and then incubated for $45 \mathrm{~min}$ with $100 \mu \mathrm{l}$ of anti-mouse IgG diluted $1: 10000$ in $0.1 \% \mathrm{BSA} / \mathrm{PBS}$ at $37^{\circ} \mathrm{C}$, washed again, and detected with $100 \mu \mathrm{l}$ of 3,3',5,5' - tetramethyl benzidine (TMB) for $20 \mathrm{~min}$ at room temprature $(\mathrm{RT})$. The reaction was then stopped by the addition of $35 \mu \mathrm{l}$ of $2 \mathrm{M} \mathrm{H}_{2} \mathrm{SO}_{4}$. The optical density (OD) value was read at $450 \mathrm{~nm}$, using a BioRad model 860 plate reader (Bio-Rad, CA, USA).

Checker board method was used to determine the optimal antigen coating concentration, serum dilutions and HRP labeled rabbit anti-mouse $\operatorname{IgG}$ dilutions. For determine the cut-off value, twenty-four sera samples from mice uninfected with $A$. pleuropneumoniae were used as negative sera, the cut-off value was calculated using the formula: mean of the negative serum values plus three standard deviations (SDs). To determine the specificity of this method, rabbit antisera of HCV, PRRSV and PM (obtained from Dr. Qigui Yan, Sichuan Agricultural University, China) were detected 
according to the method above, except that the second antibody was anti-rabbit IgG (Zhongshan, Beijing, China) diluted $1: 10000$ in $0.1 \%$ BSA/PBS. Finally, the method was used to detect the sera of inactivated $A$. pleuropneumoniae immunized and live A. pleuropneumoniae infected mice.

\section{RESULTS}

\section{Construction of an apxIVA Prokaryotic Expression} Vector

The apxIVA gene was isolated from $A$. pleuropneumoniae ATCC 263 by PCR as described in Materials and Methods. The oligonucleotides used in the PCR reaction were designed to allow the $1152 \mathrm{bp}$ long fragment in 5' terminal part of apxIVA gene to be cloned into pET-32a(+) in frame with the poly-His purification signal. PCR and RE analysis showed that prokaryotic expression plasmid PET32a-apxIVA was successfully constructed. (Fig. 1).

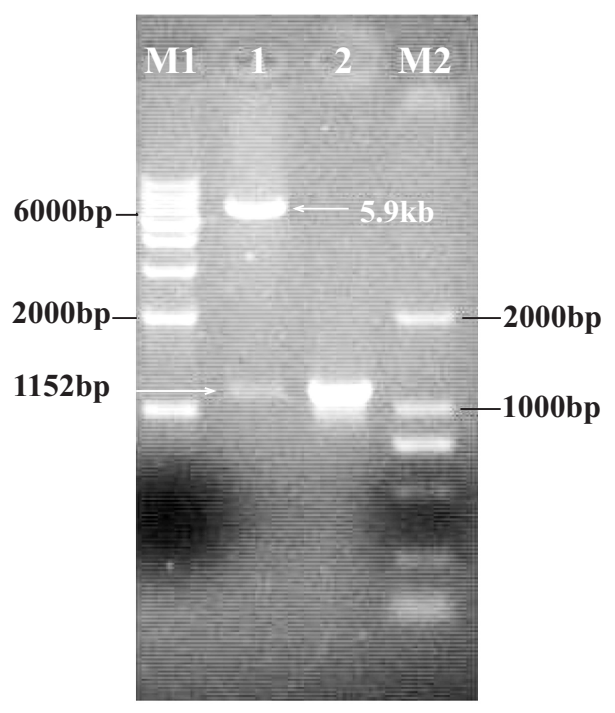

Fig. (1). Results of construction of an ApxIVa prokaryotic expression vector. M1: DNA Marker DL10000; Lane 1: prokaryotic expression vector digested with EcoR I and Xho I; Lane 2: PCR products of ApxIVa gene; M2 : DNA Marker DL2000

\section{Prokaryotic Expression and Purification of apx $I V A$}

Prokaryotic expression vector PET32a-apxIVA was induced by addition of IPTG to produce recombinant apxIVA toxin at different time ranged from $0,2,3,4,5$ to $6 \mathrm{~h}$ and at different IPTG concentration ranged from $0,0.2,0.4,0.6$, $0.8,1.0$ to $1.2 \mathrm{mmol} \mathrm{L}^{-1}$, the results showed that a protein about $62 \mathrm{Kd}$ was expressed (Fig. 2) since $1 \mathrm{~h}$ and stabilized on $4 \mathrm{~h}$ post culturation at a most appropriate IPTG concentration of $0.6 \mathrm{mM}$ (data not show ). The recombinant apxIVA was expressed mainly in the form of inclusion body and Ni-NTA superflow was used to purify this his-tagged apxIVA. For the purified protein, a single objective band was detected by SDS-PAGE (Fig. 2).

\section{Western Blot Assay}

To confirm the reactivity of his-tagged apxIVA with antibodies from $A$. pleuropneumoniae infection, the purified protein was exposed to a Western blot assay by using serum from $A$. pleuropneumoniae. The result showed that the fusion protein can react positively with serum from live $A$. pleuropneumoniae infection (Fig. 2), while negatively with serum from inactivated $A$. pleuropneumoniae immunization.(data not show).

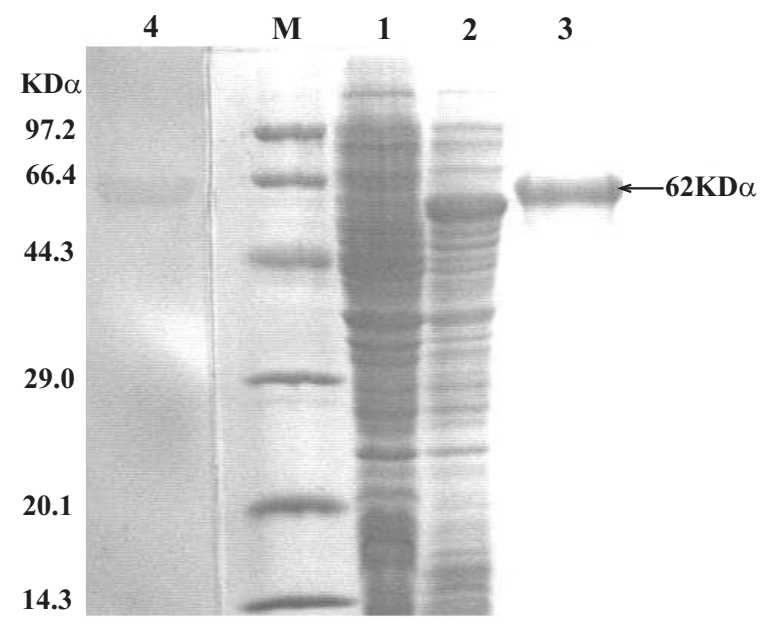

Fig. (2). Results of expression and purification and western-blot of recombinant protein. Lane M: protein marker; Lane 1: uninduced BL21 containing recombinant plasmid; Lane2: bacteria induced for 4h; Lane 3: purified Apx IVA; lane 4: western-blot result of purified recombinant protein with live $A$. pleuropneumoniae infected antisera

\section{Indirect ELISA Based on Recombinant ApxIVA}

The results of checker board experiment showed that a concentration of $1.0 \mu \mathrm{g} / \mathrm{ml}$ of apxIVA-His peptide and 1:40 serum produced the best resolution of the positive reference serum from the negative reference serum. Then 24 negative mice sera was selected randomly to react with apxIVA-His peptide, the average $\chi$ was 0.143 , standard deviation(SD) was 0.028 . The positive critical value was $\chi+3 \mathrm{SD}=0.226$. Means that while the OD value of sample $\geqslant 0.226$, the result was positive; while the OD value of sample $<0.226$, the result was negative. For the results of specificity test, positive sera of $\mathrm{HCV}$, PRRSV and Pm were negative in the apxIVA-ELISA. indicating the specificity of this apxIVAELISA (Table 1).

Table 1. The Results of Specificity Test of apxIVA-ELISA

\begin{tabular}{|c|c|c|c|c|}
\hline \multirow{4}{*}{$\begin{array}{c}\text { OD }_{450} \text { of } \\
\text { apxIVa- ELISA }\end{array}$} & \multicolumn{4}{|c|}{ Positive Sera } \\
\cline { 2 - 5 } & Sera Number & PRRSV & HCV & Pm \\
\cline { 2 - 5 } & 1 & 0.151 & 0.083 & 0.105 \\
\cline { 2 - 5 } & 2 & 0.095 & 0.162 & 0.178 \\
\cline { 2 - 5 } & 3 & 0.133 & 0.098 & 0.135 \\
\hline
\end{tabular}
virus; Pm: Pasteurella multocida.

For the results of discrimination of infection and inactivated vaccine immunization, Sera of four mice immunized with inactivated $A$. pleuropneumoniae, four mice 
Table 2. Detection of Antibodies Against A. pleuropneumoniae Infection and Inactivated A. pleuropneumoniae Immunization

\begin{tabular}{|c|c|c|c|c|c|}
\hline \multicolumn{9}{|c|}{ Serum } \\
\hline & $\begin{array}{c}\text { Animal } \\
\text { Numbers }\end{array}$ & $\begin{array}{c}\text { Inactivated App Serotype 5 } \\
\text { Vaccinated Mice }\end{array}$ & $\begin{array}{c}\text { live App Serotype 1 } \\
\text { Infected Mice }\end{array}$ & $\begin{array}{c}\text { Live App Serotype 5 } \\
\text { Infected Mice }\end{array}$ & $\begin{array}{c}\text { Live App Serotype 7 } \\
\text { Infected Mice }\end{array}$ \\
\cline { 2 - 7 } & 1 & 0.171 & 1.145 & 1.297 & 1.175 \\
\hline \multirow{3}{*}{ OD $_{450}$ of apxIva-ELISA } & 2 & 0.133 & 0.899 & 1.183 & 1.215 \\
\cline { 2 - 7 } & 3 & 0.201 & 1.013 & 1.033 & 0.933 \\
\cline { 2 - 7 } & 4 & 0.145 & 1.235 & & \\
\hline
\end{tabular}

App: A. pleuropneumoniae.

infected with live serotype 1, four mice infected with live serotype 5 and three mice infected with live serotype $7 \mathrm{~A}$. pleuropneumoniae were analyzed by apxIVA-ELISA. The results showed that all the sera of mice immunized with inactivated $A$. pleuropneumoniae were negative in the apxIVA-ELISA, while all the mice infected with live serotype 1 or 5 or 7 A. pleuropneumoniae gave positive results (Table 2 ).

\section{DISCUSSION}

Porcine pleuropneumonia was an respiratory disease hazardous to China pig industry. The virulence of the 15 serotypes of $A$. pleuropneumoniae is mainly determined by three major RTX toxins apx I, apx II and apx III [7], which are secreted by the different serotypes in various combinations, but apxIV can be produced by all 15 serotypes only during infection, but not under in vitro conditions [4].

The diagnosis of $A$. pleuropneumoniae infections is important for the identification of farms that are latently infected with A. pleuropneumoniae, detection of different $A$. pleuropneumoniae serotypes, inspection of the animals on their importation into a farm, or eradication of the infection. It is done routinely by serological examination of pig herds or by bacterial agent cultivation from nasal swabs or lungs with pleuropneumonia lesions or by PCR. Serological examination were usually carried out by detecting antibodies provoked by Apx toxin or outer membrane protein (OMP) or lipopolysaccharides (LPS) etc. OMP are common to all $A$. pleuropneumoniae serotypes [8], while LPS are serotypespecific and used to serologically distinguish infections with different serotypes of A. pleuropneumoniae [9], LPS was also found to give serological cross-reaction with nonpathogenic Actinobacillus species [10]. PCR-based methods were suitable for the rapid detection of $A$. pleuropneumoniae [11].

Vaccination is potentially an effective tool for the control of Porcine pleuropneumonia, although natural or experimental infection generally elicits at least partially protection against reinfection with another serotype [12]. Killed whole cell bacterins were commercially used in China now, and a major drawback of vaccination, is that traditional serological tests including hemmaglutination test $[13,14]$ and ELISA [15], cannot distinguish immunization of killed whole cell bacteria from field exposition. As apxIVA can be expressed by all 15 serotypes only in vivo conditions, therefore, antibodies against apxIVA should be the target of serological differentiation between immunization with killed whole cell bacteria vaccine and infection with live $A$. pleuropneumoniae.

Although an indirect ELISA based on recombinant apxIVA of $A$. pleuropneumoniae serotype 1 have been reported previously [16], but indirect ELISA based on recombinant apxIVA of serotype 5 have not been reported now. The serotype 5 is highly pathogenic, and the sequence of apxIVA of serotype 5 was different from those of serotype 1 and 3 in length and homology [17]. So we develop an indirect ELISA based on recombinant apxIVA of serotype 5 to see whether it can also detect antibodies against $A$. pleuropneumoniae infection. After the bioinformatic analysis of apxIVA gene sequence of serotype 5 strain CVCC 263, it was found that the 5'-terminal of apxIVA gene was highly antigenic, and subsequently a $1152 \mathrm{bp}$ fragment in this region was amplified and expressed to establish an indirect ELISA for detecting the antibody against the apxIVA of all serotypes. The results showed that the sera from inactivated vaccine immunized mice were negative, while the sera from live $A$. pleuropneumoniae infected mice were positive. So this indirect ELISA could detect $A$. pleuropneumoniae infection in a mouse model. The validation of this apxIVAELISA to detect $A$. pleuropneumoniae infection in pigs will be further undertaken.

\section{ACKNOWLEDGEMENTS}

This work was financially supported by Sichuan Province Basic Research Program and Program for Changjiang Scholars and Innovative Research Team in University "PCSIRT" (Grant No: IRTO848)

\section{ABBREVIATIONS}

ELISA $=$ Enzyme-linked immunosorbent assay

HRP $=$ Horseradish peroxidase

i.p. $\quad=$ Intraperitoneally

IPTG = Isopropy $1-\beta$-D-thiogalactoside

LPS $\quad=$ Lipopolysaccharides

NAD $=$ Nicotinamide adenine dinucleotide

OD $=$ Optical density

OMP $=$ Outer membrane protein

PBS $=$ Phosphate-buffered saline

PCR $=$ Polymerase Chain Reaction 


$$
\begin{aligned}
& \text { RE } \quad=\text { Restriction enzyme } \\
& \text { RT }=\text { Room temprature } \\
& \text { SDS-PAGE }=\text { Sodium dodecyl sulfate polyacrylamide gel } \\
& \text { electrophoresis } \\
& \text { TBST }=\text { Tris-Buffered Saline with Tween } 20 \\
& \text { TSB }=\text { Tryptic Soy Broth }
\end{aligned}
$$

\section{REFERENCES}

[1] Bosse JT, Janson H, Sheehan BJ, et al. Actinobacillus pleuropneumoniae: pathobiology and pathogenesis of infection. Microbes Infect 2002; 4(2): 225-35.

[2] Blackall PJ, Klaasen HL, van den Bosch H, Kuhnert P, Frey J. Proposal of a new serovar of Actinobacillus pleuropneumoniae: serovar 15. Vet Microbiol 2002; 84(1-2): 47-52.

[3] Komal JP, Mittal KR. Grouping of Actinobacillus pleuropneumoniae strains of serotypes 1 through 12 on the basis of their virulence in mice. Vet Microbiol 1990; 25(2-3): 229-40.

[4] Schaller A, Kuhn R, Kuhnert P, et al. Characterization of apxIVA, a new RTX determinant of Actinobacillus pleuropneumoniae. Microbiology 1999; 145 (Pt 8): 2105-16.

[5] Wang C, Wang Y, Shao M, et al. Positive role for rApxIVN in the immune protection of pigs against infection by Actinobacillus pleuropneumoniae. Vaccine 2009; 27(42): 5816-21.

[6] Daban M, Medrano A, Querol E. Cloning, sequencing and expression of the transferrin-binding protein 1 gene from Actinobacillus pleuropneumoniae. Biochem J 1996; 315 (Pt 1): 257-64.

[7] Frey J. Virulence in Actinobacillus pleuropneumoniae and RTX toxins. Trends Microbiol 1995; 3(7): 257-61.

[8] Beynon LM, Griffith DW, Richards JC, Perry MB. Characterization of the lipopolysaccharide $\mathrm{O}$ antigens of Actinobacillus pleuropneumoniae serotypes 9 and 11: antigenic relationships among serotypes 9, 11, and 1. J Bacteriol 1992; 174(16): 5324-31.
[9] Mittal KR, Bourdon S, Berrouard M. Evaluation of counterimmunoelectrophoresis for serotyping Actinobacillus pleuropneumoniae isolates and detection of type-specific antigens in lungs of infected pigs. J Clin Microbiol 1993; 31(9): 2339-42.

[10] Gottschalk M, Broes A, Mittal KR, et al. Non-pathogenic Actinobacillus isolates antigenically and biochemically similar to Actinobacillus pleuropneumoniae: a novel species? Vet Microbiol 2003; 92(1-2): 87-101.

[11] Schaller A, Djordjevic SP, Eamens GJ, et al. Identification and detection of Actinobacillus pleuropneumoniae by PCR based on the gene apxIVA. Vet Microbiol 2001; 79(1): 47-62.

[12] Cruijsen T, van Leengoed LA, Ham-Hoffies M, Verheijden JH. Convalescent pigs are protected completely against infection with a homologous Actinobacillus pleuropneumoniae strain but incompletely against a heterologous-serotype strain. Infect Immun 1995; 63(6): 2341-3.

[13] Mittal KR, Higgins R, Lariviere S, Leblanc D. A 2mercaptoethanol tube agglutination test for diagnosis of Haemophilus pleuropneumoniae infection in pigs. Am J Vet Res 1984; 45(4): 715-9.

[14] Habrun B, Bilic V, Humski A. Comparison of ELISA and 2-META assays used in serological diagnosis of infection with Actinobacillus pleuropneumoniae serotypes 2 and 4-7 in breeding pigs in Croatia. Prev Vet Med 1998; 36(3): 179-86.

[15] Grondahl-Hansen J, Barfod K, Klausen J, Andresen LO, Heegaard PM, Sorensen V. Development and evaluation of a mixed longchain lipopolysaccharide based ELISA for serological surveillance of infection with Actinobacillus pleuropneumoniae serotypes 2, 6 and 12 in pig herds. Vet Microbiol 2003; 96(1): 41-51.

[16] Dreyfus A, Schaller A, Nivollet S, et al. Use of recombinant ApxIV in serodiagnosis of Actinobacillus pleuropneumoniae infections, development and prevalidation of the ApxIV ELISA. Vet Microbiol 2004; 99(3-4): 227-38.

[17] Foote SJ, Bosse JT, Bouevitch AB, Langford PR, Young NM, Nash JH. The complete genome sequence of Actinobacillus pleuropneumoniae L20 (serotype 5b). J Bacteriol 2008; 190(4): 1495-6.

This is an open access article licensed under the terms of the Creative Commons Attribution Non-Commercial License (http://creativecommons.org/licenses/by-nc/ 3.0/) which permits unrestricted, non-commercial use, distribution and reproduction in any medium, provided the work is properly cited. 